\title{
Hospitalization and mortality of HIV patients in a low resources setting
}

\author{
Martin Hojma*, Paula Nada, Saldaño Ana Gomez and Maria Laura Ordoñez
}

Address: Hospital Carrillo - Los Polvorines, Buenos Aires, Argentina

* Corresponding author

from 2006 International Meeting of The Institute of Human Virology

Baltimore, USA. 17-2I November, 2006

Published: 21 December 2006

Retrovirology 2006, 3(SuppI I):P26 doi:I0.I I86/I742-4690-3-SI-P26

(C) 2006 Hojma et al; licensee BioMed Central Ltd.

\section{Introduction}

Morbi-mortality of HIV patients in HAART-era in resource-limited settings has not been well described.

\section{Objective}

To describe causes of serious complications leading to hospitalization and death in these patients.

\section{Methods}

Prospective follow up of all HIV positive patients admitted during 2004 in our Hospital, regarding sex, gender, time of HIV diagnosis, administration of HAART, viral load and CD4 count at the moment of hospitalization and outcome.

\section{Results}

Throughout the study period, there were a total of 28 hospitalizations of HIV positive patients. $17(60.7 \%)$ were men. Mean age was 35.28 yrs. Mean CD4 cell count was $108.5 \mathrm{cell} / \mu \mathrm{L}$, mean viral load 244781 copies $/ \mathrm{mL}$. Causes of hospitalization and were: 7 CNS cryptococcosis $(25 \%)$, 5 community acquired pneumonia (17.8\%), 4 CNS toxoplasmosis $(14.3 \%), 2$ pulmonary TB $(7.14 \%), 2$ pleural TB $(7.14 \%), 2$ wasting syndrome $(7.14 \%), 1$ CNS TB (3.5\%), 1 CMV retinopathy $(3.5 \%), 1$ progressive multifocal leukoencephalopathy $(3,5 \%), 1$ Kaposi's sarcoma $(3,5 \%), 2$ unknown diagnosis $(7.14 \%)$. In 5 cases (17, $8 \%)$, HIV diagnosis was made during hospitalization (2 CNS cryptococcosis, 2 community acquired pneumonia, 1 pulmonary TB). 11 patients $(39,3 \%)$ were already on HAART. Twelve patients died $(42.85 \%)$. Causes of death were 5 CNS crytococcosis $(41.6 \%), 1$ community

acquired pneumonia $(8.4 \%), 1 \mathrm{CNS}$ toxoplasmosis $(8$, $4 \%), 1$ pleural TB $(8.4 \%)(7,14 \%), 1$ CNS TB $(8,4 \%), 1$ progressive multifocal leukoencephalopathy $(8.4 \%), 1$ Kaposi's sarcoma (8.4\%), 1 unknown diagnosis (8.4\%).

\section{Conclusion}

Causes of hospital admission were multiple in HIV patients. CNS cryptococcosis was the most common cause of hospitalization and death. There was a high death rate, mostly related to crytococcosis. This could be related to social and economical factors in a low resources setting. 